\title{
视觉追踪训练联合六字决对帕金森病患者 运动功能和生活质量的影响
}

\author{
王万宏 ${ }^{1}$, 毕鸿雁 ${ }^{2}$, 邱振刚 ${ }^{1}$, 刘西花 ${ }^{2}$, 庄 贺 ${ }^{1}$, 郝世杰 ${ }^{1}$, 张 鍂 $^{*}{ }^{*}$, 孙文王 ${ }^{1}$ \\ 1 山东中医药大学, 山东 济南 250355 ; \\ 2 山东中医药大学附属医院,山东济南 250014 \\ *通信作者: 张嚅, E-mail: sdutcmkf@163.com
}

收稿日期: 2020-08-10; 接受日期 : 2020-09-30

项目基金: 山东省中医药科技发展计划项目 (2019-0167); 国家自然科学基金青年项目 (81802239)

DOI : $10.3724 /$ SP.J.1329.2020.06010

开放科学 (资源服务)标识码 (OSID)：

摘要 目的:观察视觉追踪训练联合六字诀对帕金森病患者运动功能和生活质量的影响。方法: 选择在山东 中医药大学附属医院治疗的帕金森病患者 42 例, 按照随机数字表法分为对照组和观察组, 每组 21 例。对照 组接受常规康复训练, 主要包括核心训练、平衡训练、协调性训练、步态训练、日常生活能力训练, 每天上、下 午各 1 次, 每次 $45 \mathrm{~min}$, 每周 $5 \mathrm{~d}$, 持续训练 6 周。观察组在对照组基础上接受视觉追踪联合六字诀训练。视 觉追踪训练包括: 拇指追踪、打地鼠游戏、姿势控制以及眼球转“8”字等训练, 每次训练 $20 \mathrm{~min}$, 每天 1 次; 六 字诀训绕主要色括吹、呼、嘻、呵、嘘、呬等呼吸训练, 配合肢体导引训练, 每次训练 $25 \mathrm{~min}$, 每天 1 次。以上训 练每周 $5 \mathrm{~d}$, 持续训练 6 周。采用统一的帕金森病评分量表第三部分运动分量表(UPDRS-III) 和起身一步行 计时测试 (TUGT) 对患者运动功能进行评定; 采用平衡稳定极限测试 (AP-SD、ML-SD) 对患者平衡功能进 行评估; 采用帕金森患者生活质量评定量表( PDQ-39) 对患者生活质量进行评估。结果: 治疗前, 2 组 UPDRS-III评分、AP-SD 和 ML-SD、TUGT 时间和 PDQ-39 评分无明显区别, 差异无统计学意义 $(P>0.05)$; 与 治疗前比较, 2 组治疗后 UPDRS-III、AP-SD、ML-SD、TUGT 和 PDQ-39 评分均明显降低, 差异具有统计学 意义 $(P<0.05)$; 与对照组比较, 观察组 UPDRS-III、AP-SD、ML-SD、TUGT 和 PDQ-39 评分均明显更低, 差异具有统计学意义 $(P<0.05)$ 。结论: 视觉追踪训练联合六字诀可以有效改善帕金森病患者的运动功能和 生活质量。

关键词帕金森病; 视觉追踪训练; 六字块; 运动功能; 生活质量

帕金森病 (Parkinson's disease, PD) 是一种常见 的退行性神经疾病, 其临床症状以静止性震颤、肌 肉强直、运动性迟缓、冻结步态等运动功能障碍为 主, 同时还可伴有不同程度的焦虑抑郁、睡眠障碍 以及认知障碍等非运动症状 ${ }^{[1]}$ 。据统计, 我国帕金森 病患者数量居世界首位, 已经超过 260 万, 且每年 以 $797 / 10$ 万的速度增长 ${ }^{[2] 。}$

对于帕金森病患者的治疗, 目前仍以药物、手术 治疗为主 ${ }^{[3]}$ 。药物治疗容易出现一系列并发症。手术 费用昂贵, 且效果不是很理想。帕金森病患者随着
疾病进展, 其呼吸功能常出现严重的功能障碍, 如: 呼吸肌无力、上呼吸道阻塞、肺功能下降以及睡眠 呼吸失调等症状。有研究显示, 传统功法䥿炼可以 有效改善帕金森病患者运动功能、平衡能力以及呼 吸功能 ${ }^{[4]}$, 而六字决是我国传统的养生功法, 其强调 通过呼吸训练配合导引术, 充分诱发和调动脏腑的 潜在能力来抵御疾病的侵袭, 可有效改善帕金森患 者症状。此外, 本课题组前期研究证实视觉追踪训 练可有效改善脑卒中患者的运动功能和平衡能力 ${ }^{[5]}$ 。 本研究尝试采用视觉追踪训练联合六字诀进行治

引用格式:王万宏, 毕鸿雁, 邱振刚, 等. 视觉追踪训练联合六字诀对㕷金森病患者运动功能和生活质量的影响 $[\mathbf{J}]$. 康复学报, 2020,30(6):474-478.

WANG W H, BI H Y, OIU Z G, et al. Effect of visual tracking training combined with six-character formula on motor function and quality of life in patients with Parkinson’s disease [J]. Rehabilitation Medicine, 2020,30(6):474-478.

DOI: $10.3724 /$ SP.J.1329.2020.06010 
疗,取得良好疗效。现报道如下。

\section{1 临床资料}

\section{1 病例选择标准}

1.1.1 诊断标准 符合 2016 版《中国帕金森病的诊 断标准》中关于帕金森病的诊断标准 ${ }^{[6]}$ 。

1.1.2 纳入标准 (1) 年龄: $45 \sim 81$ 岁; (2) 病程> 3 个月; (3) 帕金森 Hoehn-Yahr(修正) 分级 1 3 级, 且伴有震颤症状; (4) 眼球运动功能正常, 无视听觉 障碍; (5) 同意参加本研究,并自愿签署知情同意书。

1.1.3 排除标准 (1) 严重的心、脑、肝、肾等脏器功 能减退或衰竭者; (2) 有认知功能障碍者; (3) 伴有其 他严重神经系统疾病者。

1.1.4 中止和脱落标准 (1) 患者或家属治疗依从
性不高, 无法配合本研究方案实施; (2) 治疗过程中, 擅自接受可能影响疗效评价的其他疗法; (3) 治疗过 程中, 病情恶化或出现其他并发症无法继续接受观 察。

\section{2 一般资料}

选取 2019 年 4 月一-2020 年 8 月在山东中医药 大学附属医院康复科治疗的帕金森病患者 42 例, 按照随机数字表法分为对照组和观察组,每组 21 例。 治疗期间, 观察组 1 例患者因出现并发症无法继续 接受观察而脱落, 最终纳人 20 例。2 组性别、年龄、 病程、Hoehn-Yahr 分级等一般资料比较, 差异均无 统计学意义 $(P>0.05)$, 具有可比性, 见表 1 。本研究 经山东中医药大学附属医院伦理委员会审核通过 [批件号: [2019]伦审第(054)号-KY]。

表 12 组一般资料比较 $(\bar{x} \pm s)$

Table 1 Comparison of general data between the two groups $(\bar{x} \pm s)$

\begin{tabular}{|c|c|c|c|c|c|c|c|c|c|}
\hline \multirow{2}{*}{ 组 别 } & \multirow{2}{*}{$n$} & \multicolumn{2}{|c|}{ 性别 } & \multirow{2}{*}{ 年龄/岁 } & \multirow{2}{*}{ 病程/年 } & \multicolumn{4}{|c|}{ Hoehn-Yahr 分级 } \\
\hline & & 男 & 女 & & & 1.5 级 & 2 级 & 2.5 级 & 3 级 \\
\hline 对照组 & 21 & 11 & 10 & $64.64 \pm 7.43$ & $6.57 \pm 3.43$ & 3 & 7 & 8 & 3 \\
\hline 观察组 & 20 & 12 & 8 & $64.23 \pm 5.14$ & $7.14 \pm 3.57$ & 3 & 9 & 6 & 2 \\
\hline
\end{tabular}

\section{2 方 法}

\section{1 治疗方法}

2 组均接受药物治疗和健康教育。健康教育内 容包括:安全用药、合理饮食、帕金森病知识教育以 及日常生活安全措施教育等。

2.1.1 对照组 在《中国帕金森病的诊断标准 $(2016$ 版) 》[6]指导规律用药基础上进行常规的康复训练。 康复项目主要是针对帕金森病患者运动障碍和非 运动症状进行康复,包括核心训练、平衡训练、协调 性训练、步态训练以及日常生活能力训练等 ${ }^{[7]}$, 每次 训练 $45 \mathrm{~min}$, 每天上、下午各训练 1 次, 每周 $5 \mathrm{~d}$, 共训 练 6 周。

2.1.2 观察组 在对照组基础上给予视觉追踪训 练联合六字诀训练。

2.1.2.1 视觉追踪训练 视党追踪训练主要包括拇 指追踪训练、打地鼠游戏训练、姿势控制训练、眼球 转 “ 8 ” 字训练 ${ }^{[5]}$ 。每次训练 $20 \mathrm{~min}$, 每天 1 次, 每周 $5 \mathrm{~d}$, 共训练 6 周。

(1) 拇指追踪训练 训练者与患者距离 $40 \mathrm{~cm}$ 相对而坐, 嘱患者双眼紧盯训练者印堂穴, 训练者 把拇指䞨起, 放在印堂穴前方, 嘱患者保持头部不 动, 让双眼跟踪训练者的拇指进行左右、上下、对角
线以及旋转等方向运动。 $1 \mathrm{~min} /$ 次, 每天训练 3 次。 (2) 打地鼠游戏训练 准备 1 个纸质良好的鞋 盒, 剪掉对称 2 条长边, 保留 2 条短边作为支撑; 在 盒面上画 6 个大小一样的圆, 并剪下来, 用纸粘住; 用彩泥制作地鼠粘在纸做的棍子上当作地鼠; 团 1 个纸球粘在一次性筷子上做打棒。训练方法: 训练 者随机让地鼠出现在洞口, 患者头不旋转, 通过眼 球追踪出现在洞口的地鼠, 进行打地鼠游戏。10 次 为 1 组,每天训练 2 组, 2 组间休息 $15 \mathrm{~s}$ 。

(3)姿势控制训练在训练大厅镜子前, 画一 条直线, 让患者双眼注视镜子, 从 $3 \mathrm{~m}$ 远处沿着所 画直线行走, 行走过程中, 保持头部中立位。3 次为 1 组, 每天训练 3 组, 2 组间休息 $15 \mathrm{~s}$ 。

(4) 眼球转 “ 8 ”字训练 患者坐位或平躺, 闭上 双眼, 保持头部中立位, 双眼做 “ 8 ”字运动。 8 次为 1 组,每天训练 5 组, 2 组问休息 $10 \mathrm{~s}$ 。

2.1.2.2 六字诀训练让患者默念“嘘、呵、呼、呬、 吹、嘻”六字不同发音, 同时配合导引动作 ${ }^{[8]}$ 。每次训 练 $25 \mathrm{~min}$, 每天 1 次, 每周 $5 \mathrm{~d}$, 共训练 6 周。

(1) “嘘”字决患者两掌心向上, 小指靠腰际， 双上肢向后收至腰间, 双脚立稳, 身体向左转 $90^{\circ}$, 同 时患者的右掌从腰间向左侧托出, 此时配合吐“嘘” 字音, 最后沿原路收回腰间。另一侧做相同的动作。 
左右两侧交替穿掌各 3 次。

(2) “呵”字诀 患者双掌心呈 $45^{\circ}$ 向下放于腰 间, 双膝微屈, 双掌心合拢由 $45^{\circ}$ 向下, 然后向前上 方如“捧月”状穿出, 到鼻尖前方时, 双手掌背相拢, 向下推, 同时吐“呵”字音,重复 6 遍。

(3) “呼”字诀 患者站立, 手心向腹, 十指相对 平放于腹前, 然后双手如“抱桶”状向前托出, 同时 配合吐“呼”音,再回起始动作, 重复 6 遍。

(4) “哯”字决患者站立, 双手相对掌心向上, 放于下腹壁, 双掌相对向上托起, 到胸前变掌, 头后 仰, 然后掌向前托出, 头由后仰恢复到中立位, 配合 吐“呬”字音,重复 6 遍。

(5) “吹”字诀 患者站立, 双手放于腰背, 然后 双手向鸟展翅一样展开, 双手掌心向内合抱如“桶” 状, 同时配合吐“吹”字音,重复 6 遍。

(6) “嘻”字诀患者站立, 双手从身体两侧托 起在胸前交叉, 双臂外旋掌心向外, 向头部方向指 尖相对托起, 同时配合吐“嘻”字音, 重复 6 遍。

\section{2 观察指标}

2 组在治疗前后均由同一治疗师 (工作经验 > 3 年, 且未参加以上治疗) 分别完成以下观察指标评 估。

2.2.1 运动功能评分 采用统一的帕金森病评分量 表 (unified Parkinson's disease rating scale, UPDRS) 的第三部分运动分量表(UPDRS- III) 进行运动功能 评定 ${ }^{[9]}$ 。共包括 14 项, 每项 $0 \sim 4$ 分, 得分越高则表 示患者的运动功能越差。

2.2.2 平衡稳定极限测试 采用 PRO-KIN 平衡仪 (意大利 TECNO-BODY 公司, 型号: PK-245) 测试 患者在睁、闭眼情况下 $30 \mathrm{~s}$ 内躯干压力中心 (centre of pressure, $\mathrm{COP}$ ) 变化, 包括 $\mathrm{COP}$ 前后移动距离标 准差 (anteroposterior standard deviation, AP-SD) 和 $\mathrm{COP}$ 左右移动距离标准差 (mediolateral standard deviation, ML-SD $)^{[10]}$ 。患者躯干压力中心位移范围 越小, 表明患者平衡功能越好。

表 32 组治疗前后 ML-SD、AP-SD 比较 $(\bar{x} \pm s)$

Table 3 Comparison of ML-SD and AP-SD between two groups before and after treatment $(\bar{x} \pm s)$

\begin{tabular}{lcccccc}
\hline \multirow{2}{*}{ 组 别 } & $n$ & \multicolumn{3}{c}{ ML-SD } & & \multicolumn{2}{c}{ AP-SD } \\
\cline { 3 - 4 } & & 治疗前 & 治疗后 & & 治疗前 & 治疗后 \\
\hline 对照组 & 21 & $71.68 \pm 11.54$ & $66.75 \pm 18.76^{1)}$ & & $78.56 \pm 17.45$ & $72.52 \pm 19.35^{1)}$ \\
观察组 & 20 & $70.74 \pm 14.76$ & $56.78 \pm 17.75^{122}$ & & $79.47 \pm 18.83$ & $61.34 \pm 18.31^{122)}$ \\
\hline
\end{tabular}

注: 与治疗前比较, 1$) P<0.05$; 与对照组比较, 2) $P<0.05$ 。

Note: Compared with before treatment, 1) $P<0.05$; Compared with the control group, 2) $P<0.05$.
2.2.3 起身-步行计时测试 采用起身-步行计时 测试 (time up and go test,TUGT) ${ }^{[11]}$ 评估患者功能性 步行能力, 测试 3 次起身-步行所需时间, 然后取平 均值。完成整个动作过程时间越短, 表明患者运动 功能越好。测试过程中被检查者不能有躯体或器械 上的辅助, 否则需重新测量。

2.2.4 日常生活质量评价 采用帕金森患者生活质 量评定量表 (the Parkinson's disease questionnaire39 , PDQ-39) 评价日常生活质量 ${ }^{[12]}$ 。主要从精神健 康、认知功能、沟通交流以及日常生活能力等 8 个 维度 (39 个问题) 评估帕金森病患者近 1 个月内日 常生活的质量, 每项评分 $0 \sim 4$ 分。得分越高, 说明 患者日常生活质量越低。

\section{3 统计学方法}

采用 SPSS 21.0 统计软件进行数据分析, 计量 资料符合正态分布采用 $(x \pm s)$ 表示, 组内比较采用配 对样本 $t$ 检验, 组间比较采用两独立样本 $t$ 检验; 计 数资料采用 $\chi^{2}$ 检验。 $P<0.05$ 为差异具有统计学 意义。

\section{3 结 果}

\subsection{2 组治疗前后 UPDRS-III评分比较}

见表 2。

表 22 组治疗前后 UPDRS- III评分比较 $(\bar{x} \pm s)$

Table 2 Comparison of UPDRS-III score between two groups before and after treatment $(\bar{x} \pm s)$

\begin{tabular}{cccc}
\hline 组 别 & $n$ & 治疗前 & \multicolumn{1}{c}{ 治疗后 } \\
\hline 对照组 & 21 & $21.18 \pm 2.46$ & $18.85 \pm 3.72^{1)}$ \\
观察组 & 20 & $20.89 \pm 2.52$ & $16.62 \pm 3.68^{1 / 2)}$ \\
\hline
\end{tabular}

注: 与治疗前比较, 1) $P<0.05$; 与对照组比较, 2) $P<0.05$ 。

Note: Compared with before treatment, 1) $P<0.05$; Compared with the control group, 2) $P<0.05$.

\subsection{2 组治疗前后 ML-SD、AP-SD 比较} 见表 3。 


\subsection{2 组治疗前后 TUGT 时间、PDQ-39 评分比较表 4。}

表 42 组治疗前后 TUGT、PDQ-39 比较 $(\bar{x} \pm s)$

Table 4 Comparison of TUGT and PDQ-39 between two groups before and after treatment $(\bar{x} \pm s)$

\begin{tabular}{|c|c|c|c|c|c|}
\hline \multirow{2}{*}{ 组 别 } & \multirow{2}{*}{$n$} & \multicolumn{2}{|c|}{$\mathrm{TUGT} / \mathrm{s}$} & \multicolumn{2}{|c|}{$P D Q-39$ 评分 } \\
\hline & & 治疗前 & 治疗后 & 治疗前 & 治疗后 \\
\hline 对照组 & 21 & $45.63 \pm 5.64$ & $41.43 \pm 5.67^{1)}$ & $73.72 \pm 27.14$ & $68.41 \pm 27.28^{1)}$ \\
\hline 观察组 & 20 & $45.23 \pm 4.57$ & $34.64 \pm 5.34^{1 / 2)}$ & $74.62 \pm 27.35$ & $63.39 \pm 28.31^{1 / 2)}$ \\
\hline
\end{tabular}

注: 与治疗前比较, 1) $P<0.05$; 与对照组比较, 2) $P<0.05$ 。

Note: Compared with before treatment, 1) $P<0.05$; Compared with the control group, 2) $P<0.05$.

\section{4 讨 论}

\section{1 视觉追踪训练联合六字诀可有效改善帕金森 病患者步行功能和平衡能力}

帕金森病患者随着病情的不断进展,会出现不 同程度的运动性迟缓、平衡障碍及姿势控制能力下 降等症状。有研究表明,不断的运动和学习可以诱 导退行性病变部位神经再生, 这种神经可塑性是帕 金森病康复的神经基础 ${ }^{[13]}$,肌肉牵拉和平衡运动可 以刺激帕金森患者大脑神经中枢皮质功能的重新 建立。本研究结果显示, 与治疗前比较, 2 组治疗后 UPDRS- III 评分、AP-SD、ML-SD、TUGT 时间均明显 减少, 且观察组 UPDRS-III评分、AP-SD、ML-SD、 TUGT 时间均明显比对照组更低。这提示, 视觉追踪 训练联合六字诀可有效改善帕金森病患者步行功 能和平衡能力。人体的平衡需要感觉及运动系统共 同参与完成, 平衡功能对步态稳定性起着非常重要 的作用。有研究显示, 脊髓小脑不断接受大量视觉 信息输人, 经过信息整合、输出, 可以调控运动系 统, 激活脊䯕小脑, 进而稳定躯干的核心 ${ }^{[14]}$, 提高平 衡和运动能力。《欧洲帕金森病物理治疗指南》认 为, 呼吸功能虽不是核心功能, 但对帕金森病物理 康复非常有价值。膈肌和腹肌作为主要的呼吸肌, 也是重要核心肌群之一, 其在运动中可以对呼吸和 躯干的稳定性进行双重调节 ${ }^{[15]}$ 。帕金森病患者常规 平衡康复方法主要是治疗师通过手法被动或主动 训练患者平衡功能, 而忽略了视觉和呼吸配合导引 术训练对平衡功能的影响。本研究通过视觉追踪训 练强化帕金森病患者脊䯣小脑的视觉信息输人, 可 以帮助帕金森病患者在大脑中枢建立一种正反馈 机制, 帮助患者提高平衡功能和运动能力; 此外, 通 过吹、呼、嘻、呵、嘘、呬六字决训练, 配合肢体导引 训练, 可以增加膈肌和腹肌的功能, 有助于帕金森 病患者的脏腑、经络、气血等加快运行, 促进脏腑功
能恢复。六字诀训练还可以改善心肺功能, 为帕金 森病患者提供足够的血氧, 增加氧气利用率, 提高 耗氧量, 加速其体内营养物质的氧化分解, 为机体 康复运动提供能量 ${ }^{[16]}$ 。

\section{2 视觉追踪训练联合六字诀可有效改善帕金森 病患者生活质量}

生活质量是指人们在健康、卫生、精神以及物 质方面对生活的满意状态。对于帕金森病患者而 言, 健康和精神方面的追求, 是改善他们生活质量 的关键。帕金森病患者除有运动、平衡、呼吸、睡眠 障碍外, 可能还伴一定程度的焦虑和抑郁。其中, 运 动和平衡功能障碍是限制生活质量的关键因素。本 研究结果显示, 与治疗前比较, 2 组治疗后 PDQ-39 评分明显降低, 观察组 PDQ-39 评分比对照组明显 更低。这提示, 视觉追踪训练联合六字决可有效改。 善帕金森病患者生活质量。在视觉追踪训练过程 中, 帕金森病患者的上行感觉系统、下行运动系统 及核心的控制被不断地强化和整合。患者的姿势控 制, 特别是核心控制能力提高后, 对促进步行和生 活功能的恢复有重要意义。这与 HARVEY 等 ${ }^{[17]}$ 研 究结果一致。此外, 六字诀训练通过呼吸吐纳可有 效激活帕金森病患者机体的迷走神经, 迷走神经兴 奋可以改善患者的焦虑、抑郁以及睡眠障碍 ${ }^{[18]}$, 进 而改善患者日常活动的能力。

\section{5 小 结}

视觉追踪训练联合六字诀可以有效改善帕金 森病患者运动功能和生活质量。但本研究还存在样 本量较小, 未对患者进行出院后随访等不足之处, 下一步研究还需要开展大样本随机对照试验, 并加 强出院后随访, 以明确其确切疗效, 为帕金森病等 神经系统疾病康复提供可借鉴的依据。

\section{参考文献}

[1] BHATTACHARYYA K B, ROSA-GRILO M. Sexual dysfunctions 
in Parkinson's disease : an underrated problem in a much discussed disorder [J]. Int Rev Neurobiol , 2017, 134:859-876.

[2]吴芉, 吴佳佳, 邱文琪, 等. 帕金森伴抑郁的中医证候及神经 可塑性机制研究进展 $[\mathrm{J}]$. 世界科学技术-中医药现代化, 2018, 20(11): 1964-1968.

[3]管细红, 朱晓钢, 刘建民, 等. 瑜伽运动对早期帕金森病人平 衡能力及害怕跌倒的影响 [J]. 护理研究, 2017,31(10): 12741276.

[4] 陈生弟.中国帕金森病治疗指南 (第三版) $[\mathrm{C}] / /$ 江西省中西 医结合学会神经科专业委员会. 江西省第七次中西医结合神 经科学术交流会论文集. 南昌: 江西省中西医结合学会神经 科专业委员会, $2015: 4$.

[5] 孙文玉, 毕鸿雁. 视觉追踪训练对脑卒中患者步行功能与日 常生活能力的影响 $[\mathrm{J}]$. 护理学报, 2020,27(7):7-10.

[6] 中华医学会神经病学分会帕金森病及运动障碍学组, 中国医 师协会神经内科医师分会帕金森病及运动障碍专业委员会. 中国帕金森病的诊断标准 (2016 版) [J]. 中华神经科杂志, $2016,49(4): 268-271$.

[7]王刚,崔海伦, 刘军,等. 帕金森病发病机制及诊断与治疗转 化研究进展 $[J]$. 中国现代神经疾病杂志, 2018,18(1):19-24.

[8] 邓丽金,张文霞,陈瀪秀. 六字诀与全身呼吸提对老年慢性阻 塞性肺疾病患者呼吸功能影响的对比研究 $[\mathrm{J}]$. 康复学报, $2018,28(3): 57-61$.

[9] KABRA A, SHARMA R, KABRA R, et al. Emerging and alternative therapies for parkinson disease: an updated review $[\mathrm{J}]$. Curr Pharm Des, 2018, 24(22):2573-2582.

[10] 孙然, 张通,赵军, 等. 㢈拟现实技术对脑卒中偏㿈患者平衡
功能的疗效 $[\mathrm{J}]$. 中国康复理论与实践, 2014, 20(5): 458-463.

[11] FOX S H, KATZENSCHLAGER R, LIM S Y, et al. International Parkinson and movement disorder society evidence-based medicine review: Update on treatments for the motor symptoms of Parkinson's disease [J]. Mov Disord, 2018, 33 (8): 1248 1266.

[12] KEDZIOR K K, KAPLAN I. Tai Chi and Parkinson's disease (PD) : a systematic overview of the scientific quality of the past systematic reviews [J]. Complement Ther Med, 2019,46:144152.

[13]方伯言. 运动帕金森病康复之“本” [N]. 健康报, 2020-04-10 (004).

[14] 起晓致,姜琳丽, 王风娇, 等, 镜像疗法结合肌电生物反馈对 脑卒中的影响 $[\mathrm{J}]$. 康复学报, $2020,30(1): 34-39,45$.

[15]王飞, 张丽娟, 史䊉, 等. 普拉提核心肌群训练治疗小脑梗死 共济失调的疗效观察 $[\mathrm{J}]$. 中华物理医学与康复杂志, 2017,39 (8) :608-610.

[16] 甘海红. 呼吸功能康复操结合六字诀呼吸法对肺心病患者气 道阻力及运动耐力的影响 $[\mathrm{J}]$. 护理实践与研究, 2020,17 (17) $: 82-84$.

[17] HARVEY M, WESTON K L, GRAY W K, et al. High-intensity interval training in people with Parkinson's disease: a randomized, controlled feasibility trial $[\mathrm{J}]$. Clin Rehabil , 2019,33(3): $428-438$.

[18] HE L, SHAO H Y, CHEN W J. Clinical progress of Parkinson's disease with depressive anxiety $[\mathrm{J}]$. Chin Med,2017,12(1): $153-156$.

\title{
Effect of Visual Tracking Training combined with Six-character Formula on Motor Function and Quality of Life in Patients with Parkinson's Disease
}

WANG Wanhong ${ }^{1}$, BI Hongyan ${ }^{2}$, QIU Zhengang ${ }^{1}$, LIU Xihua ${ }^{2}$, ZHUANG He $^{1}$, HAO Shijie ${ }^{1}$, ZHANG Xin $^{1 *}$, SUN Wenyu ${ }^{1}$

${ }^{I}$ Shandong University of Traditional Chinese Medicine, Jinan, Shandong 250355, China;

${ }^{2}$ Department of Rehabilitation, Affiliated Hospital of Shandong University of Traditional Chinese Medicine, Jinan, Shandong 250014, China

* Correspondence: ZHANG Xin, E-mail: sdutemkf@163.com

\begin{abstract}
Objective: To observe the effects of visual tracking training combined with six-character formula on motor function and quality of life in patients with Parkinson's disease. Methods: A total of 42 patients with Parkinson's disease treated in the affiliated hospital of Shandong university of traditional Chinese medicine were selected and divided into the control group and the observation group according to random number table method, with 21 cases in each group. The control group received routine rehabilitation training, mainly including core training, balance training, coordination training, gait training, and daily life ability training, twice a day, 45 minutes each time, five days a week, continuous training for six weeks. The observation group received visual tracking training combined with six-character formula training on the basis of the control group. Visual tracking training included thumb tracking, Whack-a-mole game, posture control, training eyeball moving as figure " 8 ", each training for 20 minutes, once a day. Six-character formula of pronunciation training with blowing, shout, hippies, oh, boo, si breathing exercises, and cooperated with body guidance training, each training for 25 minutes, once a day. The above training for five days per week, continuous training for six weeks. The third part of the unified Parkinson's disease rating scale (UPDRS-III) and the time up and go test (TUGT) were used to assess the patient's motor function; the balance stability limit test (AP-SD, ML-SD) was used to evaluate the patient's balance function; the Parkinson's questionnaire-39 (PDQ-39) was used to evaluate the patient's quality of life. Results: Before treatment, there were no statistically significant difference in UPDRS III score, AP-SD and ML-SD, TUGT time and PDQ-39 score between two groups $(P>0.05)$; Compared with before treatment, the UPDRS III, AP-SD, ML-SD, TUGT and PDQ-39 scores of the two groups after treatment decreased significantly, the differences were statistically significant $(P<0.05)$; Compared with the control group, UPDRS III, AP-SD, ML-SD, TUGT and PDQ-39 scores of the observation group after treatment were lower, the differences were statistically significant $(P<0.05)$. Conclusion: Visual tracking training combined with six-character formula can effectively improve the motor function and quality of life of patients with Parkinson's disease.
\end{abstract}

KEY WORDS Parkinson's disease; visual tracking; six-character formula; motor function; quality of life DOI : $10.3724 /$ SP.J. 1329.2020 .06010 